\title{
Sweet Potato Leaves Contain Beta Carotene and Lutein for Eye Health
}

\author{
Kepaito Mandela ${ }^{1}$, Evaire Shakave ${ }^{1}$ \\ ${ }^{1}$ Department of Biological Sciences, Mount Kenya University, Kenya
}

\begin{abstract}
This article discusses the benefits of sweet potato leaves containing beta carotene and lutein for eye health. There are some problems of eyes namely Conjunctivitis, Dry Eyes, Cataract, Glaucoma and Refractive errors (blurred vision). Treatment options include surgery, including laser and traditional eye surgery. Sweet potatoes are high in beta-carotene and anthocyanins, which are powerful antioxidants that can help prevent eyesight loss and support eye health. Beta carotene (provitamin A) and vitamin $\mathrm{C}$ are plentiful in yellow sweet potato. The beta carotene and lutein content is beneficial for eye health.
\end{abstract}

Keywords: Sweet Potato Leaves, Eye Health, Beta Carotene, Lutein

Received : November 12, 2021

Received in Revised: December 11, 2021

Accepted: December 18, 2021

\section{Introduction}

White, red/orange, and purple sweet potatoes are all varieties of sweet potatoes. Each of these tubers has a unique nutritional profile and physical properties. White yam tubers are high in crude fiber and are therefore beneficial for the efficient elimination of waste products from the body's metabolism. White sweet potato has a sandy texture in comparison to other varieties of sweet potato, tastes sweet, and has less water than other varieties of sweet potato.

Red sweet potato is a kind of sweet potato with a flesh color ranging from purple to orange or orange (Wang et al., 2018). In comparison to white sweet potatoes, red sweet potatoes have a more fluid and less gritty (sandy) feel, but are softer. The flavor is not as sweet as the white kind, despite the fact that the sugar concentration is identical. Each 100 grams of white sweet potato has $260 \mathrm{mkg}$ ( $869 \mathrm{SI}$ ) of beta-carotene, whereas golden yellow red sweet potatoes provide $2900 \mathrm{mkg}$ (9675 SI) of beta-carotene and orange red sweet potatoes contain $9900 \mathrm{mkg}$ (9900 SI) of beta-carotene (32967 SI). The deeper the orange hue, the more beta-carotene there is in the food.

Beta-carotene is the building block of vitamin A in the body. Along with beta-carotene, sweet potatoes' orange hue indicates a high concentration of lutein and zeaxanthin, two carotenoid antioxidants (Van Eck et al., 2007). Both include pigments like as chlorophyll, which is converted to vitamin A. Lutein and zeaxanthin are active chemicals that play a critical function in preventing cell death. Additionally, red sweet potatoes are high in vitamin E. Antioxidants included in red sweet potatoes have been shown to slow the rate of cell damage caused by free radicals (Shih et al., 2009). As a result, red sweet potatoes may help to prevent memory loss and senility, as well as coronary heart disease and cancer. 


\section{Literature Review}

\section{Eye health problems}

\section{Conjunctivitis}

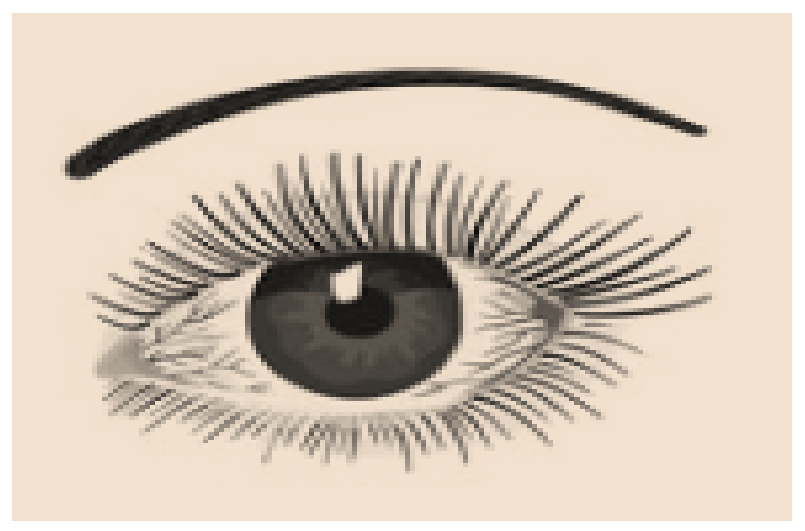

Figure 1. Conjunctivitis Eye Problem

When the soft tissue around the eye gets inflamed, the eye becomes red, watery, painful, and itchy. Conjunctivitis is a condition that can be brought on by irritation, allergies, or infection. If the infection is bacterial, the eye might get infected and fester (Jackson, 1993). This is a common cause of eye discomfort in children and adults. The treatment of conjunctivitis is determined on the etiology. If allergies are the cause of conjunctivitis, the therapy is to avoid allergens and to use antihistamines. Conjunctivitis can resolve on its own within a few days if it is caused by a viral infection. In the case of conjunctivitis caused by a bacterial infection, antibiotic eye drops or eye ointment are required.

\section{Dry Eyes}

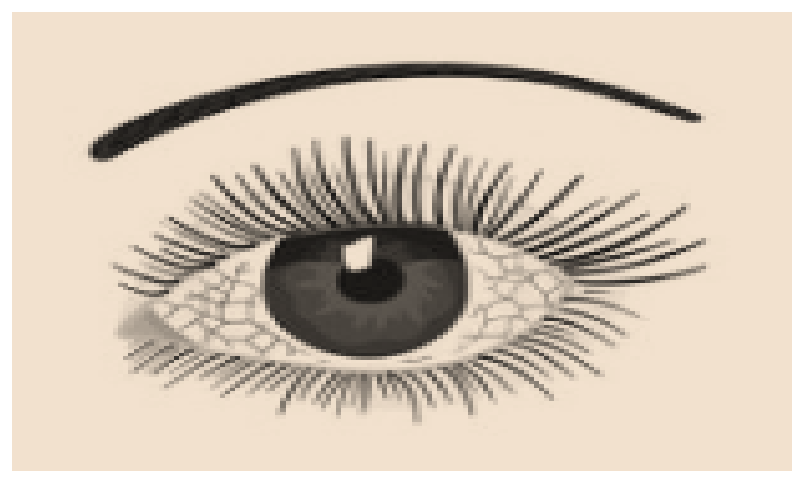

Figure 2. Dry Eyes Problem

While dry eyes can affect anybody, they are especially prevalent among the elderly and women. Dry eye symptoms include gritty eyes or the appearance of a foreign object in the eye, red eyes, burning or itching, and glare (Maskin, 2008). It is caused by a variety of circumstances, including a lack of tear production, autoimmune illnesses, infections, inflammation, allergies, dietary deficiencies, excessive exposure to wind or sunshine, and medicine side effects. Artificial tears or drugs to stimulate tear production can be used to treat dry eyes (Sharma \& Hindman, 2014). Additionally, the reasons of dry eyes must be determined and addressed. 


\section{Cataract}

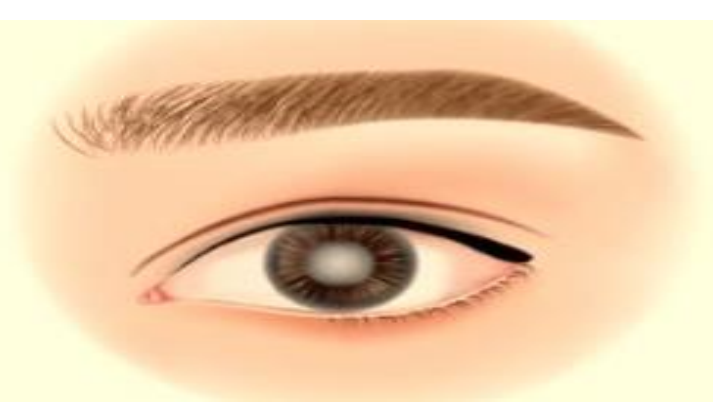

Figure 3. Cataract eye problem

Cataract is the leading cause of blindness (Steinkuller, 1983). This eye illness causes the lens of the eye to appear foggy, resulting in blurred vision. Cataracts are more common in adults over the age of 60. Cataracts, on the other hand, can develop at a younger age, even in infants. Apart from age, which results in the protein in the lens of the eye clumping together, cataracts can be induced by diabetes, eye trauma, UV exposure, smoking, and the negative effects of some drugs, such as corticosteroids and radiation treatment. Cataracts can be corrected surgically if they impair eyesight.

\section{Glaucoma}

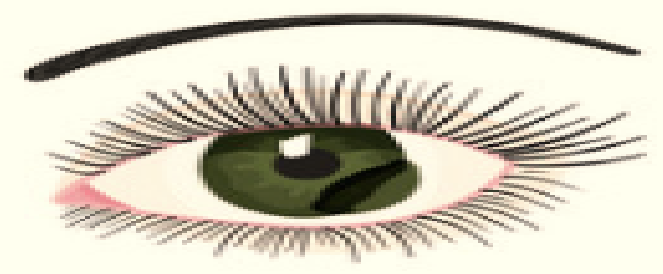

Figure 4. Glaucoma eye problem

Glaucoma affects around 6 million individuals. Glaucoma develops when the eye's optic nerve is destroyed, resulting in reduced vision and eventually blindness (Lee \& Higginbotham, 2005). Optic nerve injury occurs as a result of increased pressure in the eyeball caused by fluid accumulation. Glaucoma may strike anybody at any age, although it is more prevalent among the elderly. This eye condition is a primary cause of blindness in those over the age of 60 . Oral medicine or eye drops might be used to relieve pressure inside the eyeball. Additional treatment options include surgery, including laser and traditional eye surgery.

\section{Refractive errors (blurred vision)}

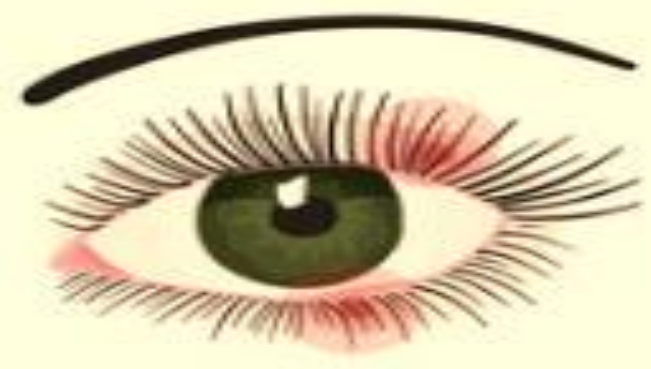

Figure 5. Refractive errors (blurred vision) eye problem 
Refractive errors impair the ability of patients to see clearly, as the eye's focus does not fall exactly where it should (Sakimoto et al., 2006). Normally, the focus of light or picture captured by the eye falls on the back of the eye, namely on the retina. Light does not concentrate perfectly on the retina in those who have refractive defects. As a result, objects become hazy. Additionally, refractive errors can be induced by changes in the cornea's shape or lens age. There are four forms of refractive errors: (1) nearsightedness, (2) farsightedness, and (3) farsightedness. Patients are unable to see close objects well due to the light's focus being beyond the retina. (4) Myopia. Faraway objects appear blurred because the focus of light is in front of the retina. Presbyopia, or old eyes, is a condition in which the eyes lose their capacity to see well as they age. This condition affects the muscles around the eyes, causing them to lose their suppleness and stiffen. Astigmatism, often known as cylinder eyes. This condition arises when the cornea or lens of the eye is not round in shape but is more convex or concave in shape. As a result, both distant and close vision will appear blurred.

\section{Sweet potato}

Sweet potatoes are high in beta-carotene and anthocyanins, both of which are powerful antioxidants that can help prevent eyesight loss and support eye health (Gurmu et al., 2014). 200 grams of cooked sweet potato with skin has more than seven times the daily recommended dose of beta-carotene for an adult. Consuming beta-carotene-rich foods, such as orange-fleshed sweet potatoes, can help avoid eye disorders such as nearsightedness and cataracts. Vitamin A is also necessary for the health of mucous membranes, particularly those lining the intestines.

Each hue of sweet potatoes has a unique combination of ingredients. Sweet potatoes come in a variety of hues, including white, purple, red, yellow, and orange. Beta carotene (provitamin A) and vitamin $\mathrm{C}$ are abundant in yellow sweet potatoes (de Carvalho et al., 2017). Additionally, purple tubers are an excellent source of vitamin $\mathrm{C}$ and beta carotene (provitamin A) Meanwhile, white-skinned sweet potatoes are deficient in or lack certain vitamins. White tubers, on the other hand, may be utilized to make flour due to their high dry matter content.

Yellow sweet potato is a kind of sweet potato with tuber flesh that is yellow, pale yellow, or yellowish white. For instance, layer 34 cultivars, South Queen 27 cultivars, Kawagoya cultivars, Cicah 16 cultivars, and Tis cultivars 5125-27.

\section{Characteristics of Sweet Potato}

Sweet potato species are numerous and include indigenous species as well as various superior variants. Sweet potatoes come in a variety of varieties, each with its own distinct qualities. There are four varieties of sweet potatoes that are extremely well-known in the community. They are as follows: (1) Puti sweet potato tubers are normally spherical, the skin is uneven, and the flesh is tougher and sweeter. White sweet potato has a pleasant scent, flavor, and cooking qualities; (2) Yellow Sweet Potato: the tuber is oval in shape, the skin surface is uneven, the flesh is orange/yellow in color, and it is softer (wet), resulting in a lower starch content of roughly 13-19 percent. The flavor is less sweet, but the vitamin A and C content is high; (3) Red Sweet Potato: the shape of the tuber is generally round, the skin surface is generally uneven, the flesh of the tuber is harder and the color is red in the center and white near the skin; the flavor is less sweet than the white sweet potato, and the skin surface is generally uneven. Red sweet potatoes contain the highest amount of vitamin A (retinol) of all sweet potatoes and are unaffected by the boiling process; additionally, the fiber found in red and purple sweet potatoes acts as a prebiotic, stimulating the growth of beneficial bacteria in the intestines, resulting in increased absorption of nutrients and cleaner intestines; (4) Purple 
Sweet Potato: the tuber shape is generally oval and the surface is small; the flesh is purple, sour, and firm; Purple sweet potatoes have several advantages over other forms of sweet potatoes, including the presence of beneficial antioxidants and a higher concentration of anthocyanin pigments than other sources such as purple cabbage, blueberries, and red maize.

\section{Sweet Potato Leaf Content}

Not only do sweet potato tubers have a plethora of beneficial nutrients, but they also include various critical components for the body. Sweet potato leaves are a good source of macro and micronutrients. Carbohydrates, proteins, and lipids are the macronutrients found in sweet potato leaves. Sweet potato leaves are heavy in carbohydrate and protein, but low in fat. Sweet potato leaves have a low unsaturated fat content. Additionally, sweet potato leaves have a relatively low cholesterol level. Niacin, calcium, iron, lutein, and xanthine are abundant in sweet potato leaves. Additionally, sweet potato leaves are a good source of fiber, vitamin A, vitamin $\mathrm{C}$, thiamine, riboflavin, vitamin B6, folic acid, magnesium, phosphorus, manganese, and potassium.

Sweet potato leaves include carbs, protein, calcium, and iron in addition to fiber. Additionally, the beta carotene and lutein content is beneficial for eye health. Additionally, these veggies contain high quantities of vitamin E, vitamin C, and vitamins B1, B2, and B6. These levels are sufficient to act as an anti-aging agent or to prevent aging.

Vitamin B6 is hailed as the 'agent' that transforms sweet potato leaves into a platelet-boosting supplement, since this vitamin plays a critical role in red blood cell metabolism. Additionally, it includes polyphenols that may help prevent heart disease. This leaf is thought to have the ability to help persons with type II diabetes control their blood sugar levels. A decoction of sweet potato leaves is used to treat diarrhea, stomach discomfort, nausea, and fever in China, Pakistan, and India. Crush the fresh leaves to treat small burns and immerse them (without heating) to alleviate the symptoms of asthmatic shortness of breath.

\section{Beta Carotene Level}

Beta carotene is a tetraterpenoid group carotenoid hydrocarbon molecule. Beta carotene is susceptible to oxidation due to the presence of double bonds. The oxidation of beta carotene is accelerated in the presence of light and metal catalysts. Oxidation occurs at random locations throughout the carbon chain that contains double bonds. Carotenoids are pigments found in plants that have a correlation with their nutritional value (Kim et al., 2010). The yellow or orange hue of yellow sweet potatoes suggests the presence of carotene. Beta carotene is advantageous to health in several ways, including preventing loss of vision due to xerophthalmia, night blindness, conjunctivitis (inflammation of the eyelids), retinopathy, cataracts, and impaired function of the retina in the back of the eye. Additionally, it has been shown to decrease the risk of prostate and pancreatic respiratory disorders. Beta carotene may also aid in the resolution of common female health problems, including irregular or normal menstruation, abnormal Pap tests, premenstrual syndrome, vaginitis, and urinary tract infections. Beta carotene may help prevent premature aging, boost immunity, and lower the risk of developing degenerative illnesses).

Beta carotene possesses physical features, including a light sensitivity that is dependent on the presence of oxygen, light, and heat, all of which act as catalysts throughout the oxidation process. Oxidation that breaks up the carotene ring group reduces carotene activity. 


\section{Beta Carotene Benefits}

Beta carotene is present in orange-yellow vegetables and fruits like sweet potatoes, pumpkin, and mangoes, as well as green vegetables like spinach and kale. Pumpkin has $1.18 \mathrm{mg}$ of beta carotene per $100 \mathrm{~g}$. Beta carotene is a naturally occurring chemical component present in a wide variety of fruits and vegetables. Is the finest source of an essential vitamin, vitamin A. Vitamin A is required for healthy vision and skin. While there are other molecules that include vitamin $\mathrm{A}$, beta carotene is the most abundant.

Beta carotene has a number of advantages, the first of which is that it acts as a precursor to vitamin A. Apart from being beneficial to the eyes, foods high in beta carotene also help prevent cancer. Beta carotene is an antioxidant that can aid in the stabilization of carbon-nucleated radicals, which may be beneficial in lowering the risk of cancer. The beta carotene content in natural foods may help lower the risk of stroke. This is due to beta carotene's ability to inhibit the formation of plaque or cholesterol deposits in the blood vessels. Additionally, beta carotene possesses analgesic (pain-relieving) and anti-inflammatory (anti-inflammatory) properties. Consuming up to 3,071.93 IU of beta carotene per kilogram of body weight has been shown to have analgesic and anti-inflammatory effects on the body.

\section{Benefits of sweet potato leaves}

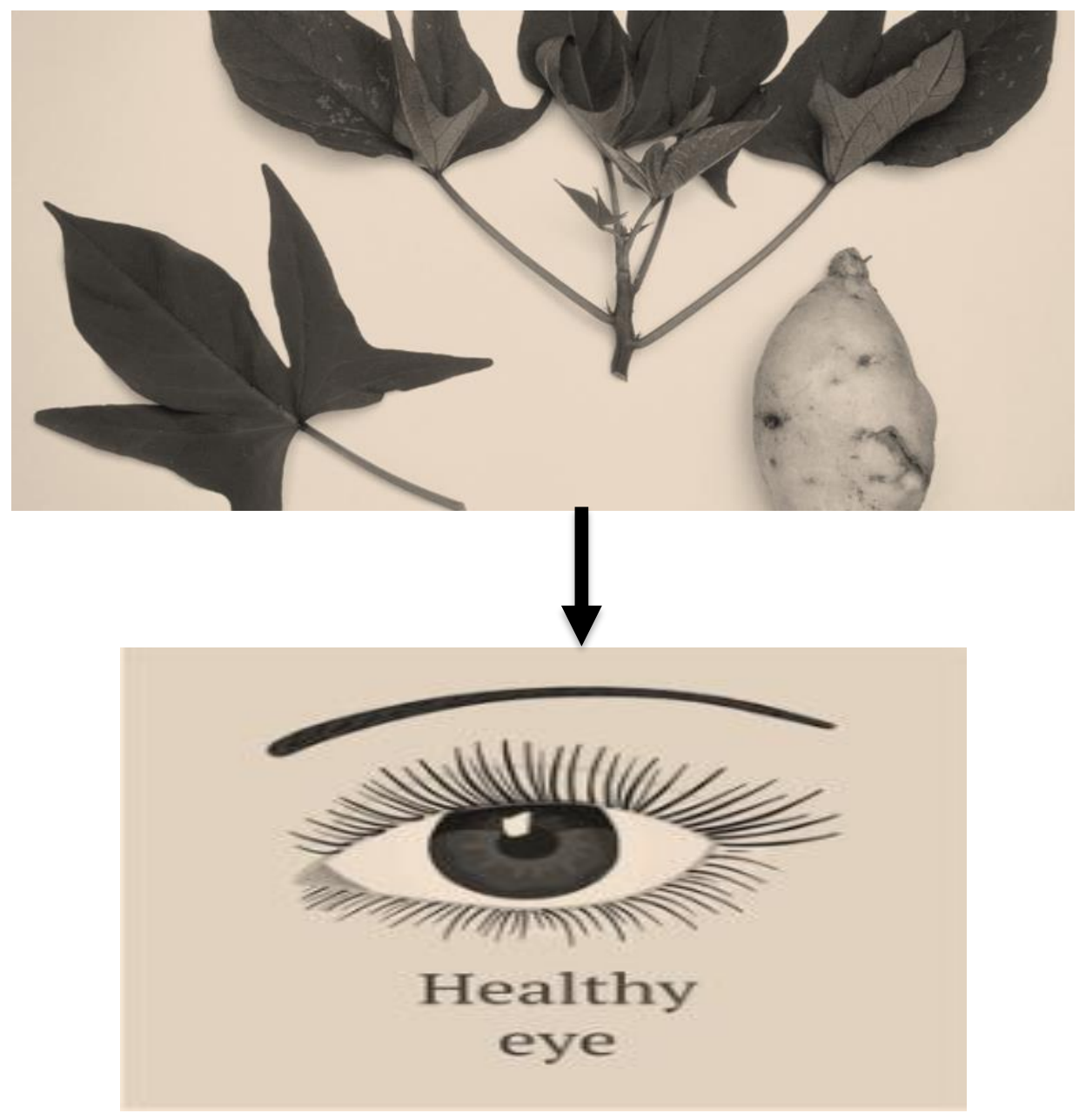

Figure 6. Sweet potato leaves for eyes problem 
The health advantages of sweet potato leaves include the following: (1) Can help lower the risk of heart disease, Sweet potato leaves have been shown to possess amazing qualities. These leaves are high in vitamin B6, which may help lessen your chance of developing heart disease. Sweet potato leaves have three times the nutritious value of other green vegetables, five times the vitamin C content, and ten times the riboflavin content; (2) Promotes bone density. Vitamin $\mathrm{K}$ found in sweet potato leaves really aids the body in retaining calcium in the bones, hence lowering the risk of developing osteoporosis. Vitamin K supplementation has been shown to reduce bone loss in osteoporosis patients. Vitamin $\mathrm{K}$ is critical for the addition of calcium during bone formation. Vitamin $\mathrm{K}$ included in sweet potato leaves may help postmenopausal women maintain healthy bones and lower their risk of fractures. Additionally, some data shows that vitamin $\mathrm{D}$ and vitamin $\mathrm{K}$ work synergistically to promote bone density. Vitamin $\mathrm{K}$ is beneficial for calcium balance. Consumption of vitamin K-rich meals following a wound or bodily harm can aid in the prevention of fracture repair; (3) Relieve menstrual pain Due to the high vitamin K content of sweet potato leaves, it is extremely beneficial for balancing hormone function and alleviating menstruation discomfort. Vitamin $\mathrm{K}$ is also beneficial for clotting blood and avoiding heavy menstrual bleeding. To be sure, if the body is vitamin $\mathrm{K}$ insufficient, cramping discomfort during menstruation will be more intense than in persons who are not vitamin K deficient; (5) Assists in increasing the strength of eyesight. Along with vitamin K, which plays a significant role in the advantages of sweet potato leaves, it turns out that they also contain vitamin A, which is just as beneficial. Vitamin A aids in the prevention of macular degeneration, the primary cause of blindness. Consumption of vitamin C, vitamin A, iron, vitamin $\mathrm{E}$, and copper found in sweet potato leaves has been shown to significantly lower the risk of macular degeneration by $25 \%$.

\section{Conclusion}

When the soft tissue around the eye gets inflamed, the eye becomes red, watery, painful, and itchy. Conjunctivitis is a condition that can be brought on by irritation, allergies, or infection. Dry eyes are caused by a variety of circumstances, including a lack of tear production and autoimmune illnesses. Glaucoma develops when the eye's optic nerve is destroyed, resulting in reduced vision and eventually blindness. Optic nerve injury occurs as a result of increased pressure in the eyeball caused by fluid accumulation. Treatment options include surgery, including laser and traditional eye surgery. Sweet potatoes are high in beta-carotene and anthocyanins, which are powerful antioxidants that can help prevent eyesight loss and support eye health. Sweet potatoes come in a variety of hues, including white, purple, red, yellow, and orange.

\section{References}

de Carvalho, L. M. J., Ortiz, G. M. D., de Carvalho, J. L. V., Smirdele, L., \& Cardoso, F. D. S. N. (2017). Carotenoids in yellow sweet potatoes, pumpkins and yellow sweet cassava. In Carotenoids. IntechOpen.

Gurmu, F., Hussein, S., \& Laing, M. (2014). The potential of orange-fleshed sweet potato to prevent vitamin A deficiency in Africa. Int J Vitam Nutr Res, 84(1-2), 65-78.

Jackson, W. B. (1993). Differentiating conjunctivitis of diverse origins. Survey of ophthalmology, 38, 91-104.

Kim, J. K., Lee, S. Y., Chu, S. M., Lim, S. H., Suh, S. C., Lee, Y. T., ... \& Ha, S. H. (2010). Variation and correlation analysis of flavonoids and carotenoids in Korean pigmented 
rice (Oryza sativa L.) cultivars. Journal of agricultural and food chemistry, 58(24), 12804-12809.

Lee, D. A., \& Higginbotham, E. J. (2005). Glaucoma and its treatment: a review. American journal of health-system pharmacy, 62(7), 691-699.

Maskin, S. L. (2008). Reversing dry eye syndrome. Yale University Press.

Sakimoto, T., Rosenblatt, M. I., \& Azar, D. T. (2006). Laser eye surgery for refractive errors. The Lancet, 367(9520), 1432-1447.

Sharma, A., \& Hindman, H. B. (2014). Aging: a predisposition to dry eyes. Journal of ophthalmology, 2014.

Shih, M. C., Kuo, C. C., \& Chiang, W. (2009). Effects of drying and extrusion on colour, chemical composition, antioxidant activities and mitogenic response of spleen lymphocytes of sweet potatoes. Food Chemistry, 117(1), 114-121.

Steinkuller, P. G. (1983). Cataract: the leading cause of blindness and vision loss in Africa. Social Science \& Medicine, 17(22), 1693-1702.

Van Eck, J. O. Y. C. E., Conlin, B. R. I. A. N., Garvin, D. F., Mason, H., Navarre, D. A., \& Brown, C. R. (2007). Enhancing beta-carotene content in potato by RNAi-mediated silencing of the beta-carotene hydroxylase gene. American Journal of Potato Research, 84(4), 331-342.

Wang, A., Li, R., Ren, L., Gao, X., Zhang, Y., Ma, Z., ... \& Luo, Y. (2018). A comparative metabolomics study of flavonoids in sweet potato with different flesh colors (Ipomoea batatas (L.) Lam). Food chemistry, 260, 124-134 